\title{
Perfil del estudiante de Licenciatura en Inglés como Lengua Extranjera, modalidad virtual
}

\author{
Nélida Cárdenas Vergaño ${ }^{1}$ \\ Clara Esperanza Bilbao ${ }^{2}$ \\ Diana Liceth Martínez ${ }^{3}$
}

\begin{abstract}
Resumen
Objetivo. Identificar y analizar las características, necesidades, motivaciones, expectativas de los estudiantes de los estudiantes de la Licenciatura en Inglés como lengua Extranjera de la Universidad Nacional Abierta y a Distancia UNAD.
\end{abstract}

Método. Etnografía en ambientes virtuales, en donde se analizan variables de tipo: demográficas, socioeconómicas, familiares, laborales, condiciones de manejo del idioma extranjero, expectativas, motivaciones intrínsecas y extrínsecas para estudiar una licenciatura en ingles con modalidad virtual.

Resultados. La mayoría de estudiantes en el rango de edad entre los 16 a 36 años, estratos 1 al 3, laborando entre 26 a 45 horas semanales, $51 \%$ con estudios previos, ingreso por gusto del aprendizaje de la lengua extranjera, con baja motivación por la docencia.

Palabras clave: Características, necesidades, motivación, expectativas, población.

2. Licenciada en Idiomas de la Universidad Nacional, Especialista en traducción español - inglés - francés de la Universidad del Rosario y Magister en Lingüística Aplicada a la Enseñanza del Español como Lengua Extranjera de la Universidad de Jaén (España). Tutora virtual de inglés de la UNAD desde el año 2007 y, a partir de 2015, Líder Nacional de la Licenciatura en Inglés como Lengua Extranjera - Distancia. Es también tutora virtual de inglés certificada por el Consejo Británico y el Ministerio de Educación Nacional.

Correo: clara.bilbao@unad.edu.co

ORCID: http://orcid.org/0000-0002-4947-9839

3. Magíster en Educación. Tutora virtual de la Licenciatura en Inglés como Lengua Extranjera de la UNAD. Co-investigadora del grupo VIRTUALEX de la ECEDU-UNAD.

Correo: diana.martinez@unad.edu.co

ORCID: http://orcid.org/0000-0002-1923-3985 


\title{
Student profile in English Bachelor degree as a Foreign Language, virtual mode
}

\begin{abstract}
Objective. Identify and analyze the characteristics, needs, motivations, expectations of students of students of the Degree in English as a foreign language of the UNAD.

Method. Ethnography in virtual environments. Where type variables are analyzed: demographic, socioeconomic, familiar, work, conditions used in foreign languages, expectations, intrinsic and extrinsic motivations to study a degree in English in virtual mode.

Results. Most students in the age range between 16 to 36 years, 1 to 3 estratification, working between $26-45$ hours per week, $51 \%$ with previous studies, affinity for learning a foreign language, with low motivation for teaching.
\end{abstract}

Keywords: Characteristics, needs, motivation, expectations, population.

\section{Introducción}

La Licenciatura en Inglés como Lengua Extranjera de la Universidad Nacional Abierta y a Distancia, es un programa que obtuvo registro calificado por parte del Ministerio de Educación Nacional a finales de abril de 2011. Desde esa fecha ha venido implementando diversas acciones con el fin de identificar las características de la población matriculada y así mismo diseñar estrategias que permitan un buen desempeño académico de los estudiantes.

Dentro de las acciones trazadas estuvo la implementación del proyecto de investigación UNAD-SIGI009, denominado Caracterización de los perfiles, 
competencias y niveles de inglés con que ingresan los estudiantes a la licenciatura en inglés como lengua extranjera de la UNAD. De acuerdo con esa denominación, los objetivos estuvieron encaminados a caracterizar los perfiles, competencias y niveles de dominio de lengua inglesa con que ingresaban los estudiantes a la Licenciatura en Inglés como Lengua Extranjera de la Universidad Nacional Abierta y a Distancia (LILE-UNAD), en los periodos académicos 2011-II, 2012-I y 2012-II, desde la identificación de los aspectos demográficos, socio-económicos, laborales y académicos, las motivaciones y expectativas de ingreso al mismo, los niveles de dominio de la lengua extranjera $\mathrm{y}$, finalmente, las condiciones y contextos de contacto con el idioma previos a la matrícula en la licenciatura. Todo lo anterior como referentes para la consolidación de estrategias que permitieran el alcance de los estándares de calidad y las competencias propuestas en el Proyecto Educativo del Programa.

Aunque inicialmente en el proyecto de investigación se propuso una metodología de tipo exploratorio/descriptivo, durante el desarrollo del mismo y gracias a la consulta permanente de los investigadores, se encontró que se acercaba bastante a una etnografía, pero en ambientes virtuales. Fue así, partiendo de lo propuesto por Hine (2000, p13):

La etnografía es una metodología ideal para iniciar estudios a internet, ya que con ella se puede explorar las complejas interrelaciones entre las tecnologías y la vida cotidiana (...). Por tanto, se percibe que la perspectiva etnográfica puede adaptarse para la comprensión de los fenómenos en internet.

Igualmente lo propuesto por Mason (2001,p35) cuando afirma que:

Una etnografía virtual es aquella que inmerge totalmente de la realidad consensuada y experienciada por grupos de personas que usan la comunicación mediada por ordenador como su primario, y a menudo único, medio de comunicación. Como tal, la persona online o virtual de los participantes es el foco principal del etnógrafo.

La metodología de investigación pasó de una simple exploración-descripción con enfoque mixto (cualitativo/cuantitativo), a una etnografía virtual en el curso introductorio de la licenciatura, donde se tuvieron en cuenta entonces, junto con la encuesta de caracterización y la prueba diagnóstica, la observación directa de las formas de interacción en el primer trabajo colaborativo y la acción comunicativa presentada en el mismo, además de revisar en detalle las respuestas dadas a las preguntas planteadas en el foro para identificar aspectos relevantes sobre las motivaciones para el curso de la carrera. 


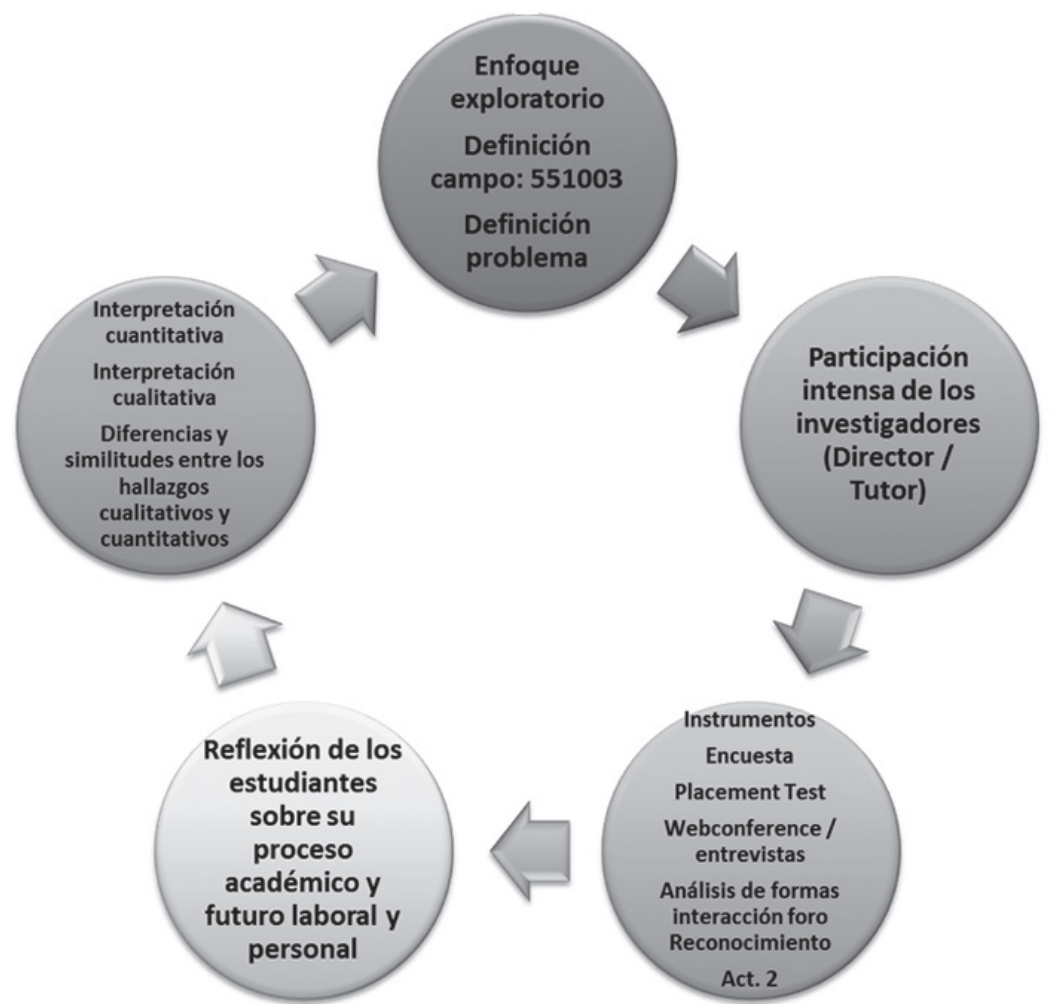

Figura 1. Metodología investigación propuesta - Etnografía (Fuente: producción propia)

\section{A modo de conceptualización}

Se hace necesario aclarar los conceptos que permitieron consolidar el proceso investigativo ya que aportaron, de manera significativa, en la planeación y ejecución del mismo.

\section{Análisis socio-demográfico}

En la investigación realizada por Américo y Lida Calero (1989) sobre el perfil del estudiante universitario, se identificaron diez aspectos básicos que agrupaban a la población estudiantil, denominados "dominios de la vida del estudiante", los cuales son: 1. Demográfico, 2. Socioeconómico, 3. Cultural, 4. Académico, 5. Cognoscitivo, 6. Socioafectivo, 7. Ético-Moral, 8. Político y gremial, 9. Salud y 10. Uso del tiempo libre. 
Sin duda, los factores sociodemográficos marcan la vida de las personas al mismo tiempo que generan características comunes a grupos sociales, lo que permite reconocer la identidad de una comunidad específica. Al analizar el aspecto socioeconómico del estudiante se aborda el estrato social del cual proviene, sus fuentes de sostenimiento y las oportunidades sociales que posee. Los aspectos demográficos juegan un papel importante en este estudio en la medida en que permiten conocer la distribución geográfica de los estudiantes de acuerdo con su lugar de origen.

En nuestro caso, el análisis socio-demográfico realizado en muestras de estudiantes de primer periodo académico, nos permitió identificar claramente diversos aspectos, con el ánimo de: 1 . Conocer los principales rasgos de la población que se interesa en nuestro Programa e ingresa a primer periodo académico, y 2. Saber si las condiciones y características actuales de nuestra Licenciatura responden de manera efectiva a sus necesidades, y de no ser así realizar los ajustes pertinentes, siempre que esto aporte al mejoramiento continuo del Programa.

\section{Competencias Comunicativas}

El término competencias comunicativas se asumió partiendo de lo planteado en los Estándares Básicos de Competencias en Lengua Extranjeras del MEN (2006), tratando de identificar el conjunto de saberes, conocimientos, destrezas y características que demostraban los estudiantes para el uso de la lengua inglesa en el momento de iniciar sus estudios en la Licenciatura en Inglés de la UNAD. Por supuesto, fue indispensable también tener en cuenta lo propuesto en el Marco Común Europeo de Referencia para las Lenguas (2006), para identificar las características de los niveles de dominio y desempeño en inglés desde nivel acceso: A1 hasta nivel de dominio eficaz: $\mathrm{C} 1$.

\section{Acción comunicativa en ambientes virtuales (formas de participación en foros).}

Por supuesto, si se habla de competencias comunicativas, es indispensable revisar también las formas de acción comunicativa que tienen los estudiantes en los cursos online del programa, desde que inician su proceso académico y que llevan al mejoramiento de ese tipo de competencias.

Barberà (citado en Barberà, Badia \& Mominó, 2001), interpreta la interacción "como un tipo de actividad sociocultural situada o como la actividad relacional y discursiva que se puede desarrollar en un determinado contexto virtual y que puede favorecer, o no, un mayor aprendizaje del estudiante" (p. 5). 
En ese mismo sentido, Barberà, Badia, \& Mominó (2001,p7), plantean

El núcleo de calidad en un contexto virtual desde tres tipos de interacciones dinámicas e interdependientes: a) entre materiales y estudiantes-profesor, b) entre estudiantes y profesor y c) entre los propios estudiantes.

Para el caso de esta investigación se tendrá en cuenta principalmente la interacción entre el estudiante-profesor y entre los propios estudiantes del grupo colaborativo. Pero no basta con reconocer si los agentes que intervienen en el contexto virtual interactúan o no, es importante también saber si la cantidad y la calidad de esas interacciones conducen a un proceso de aprendizaje y de desarrollo de competencias comunicativas por parte del estudiante y de acompañamiento significativo por parte del docente-tutor del curso introductorio a la Licenciatura en Inglés como Lengua Extranjera.

Moore y Anderson (2003), por su parte proponen que "The quality and quantity of student-teacher interaction is dependent on the instructional design and selection of learning activities developed for the instructional program"4 ( $p$. 134). Es por eso que desde el mismo diseño de un curso virtual se debe tener en cuenta el tipo de retroalimentación que requieren los foros propuestos, con el fin de orientar un verdadero intercambio comunicativo y de discusión, "una conversación didáctica guiada" (Holmberg, 1989, como se citó en Camacho et al, 2007, p. 4) mediatizada por componentes comunicacionales, pedagógicos, tecnológicos, afectivos y sociales.

En los cursos virtuales de la UNAD, el docente-tutor es el encargado de coordinar la comunicación sincrónica o asincrónica en los foros colaborativos propuestos desde el mismo diseño CORE. En palabras de Sánchez, Puerta \& Sánchez (2010), "el docente encargado de coordinar (...) se convierte en un facilitador de encuentros y de generación de conocimiento y saberes en red" (p. 30). Recordemos también que es vital que el docente-tutor reconozca el grupo, las formas de comunicación del mismo, el intercambio cultural y a la vez, reconocer las experiencias previas de sus estudiantes en ambientes virtuales de aprendizaje.

Fue prioritario entonces reconocer el tipo de interacción entre estudiantesestudiantes y estudiantes-docente durante el desarrollo de la Actividad colaborativa 1: reconocimiento, propuesta en el curso Introducción a la Licenciatura en Inglés como Lengua Extranjera. Para realizar este análisis tomamos como base las microacciones observadas por los investigadores, tal como lo proponen Claudia Camacho, Adriana Pacheco y Marco Antonio Pereida (2007) en su propuesta El análisis e interpretación de las interacciones en los foros virtuales: descripción de parámetros orientadores para mejorar la calidad realizado en el Sistema de Universidad Virtual (SUV) de la Universidad de Guadalajara (UdG). 
Es así como la acción social de la comunicación es parte del análisis de la interacción de los estudiantes y el docente-tutor en el foro de reconocimiento del curso introductorio. Vale recordar que las actividades propuestas en dicho foro buscan la identificación de los participantes en el curso, reconocer las motivaciones y expectativas de los mismos hacia el programa, además de las experiencias previas tanto en el aprendizaje del inglés como en la interacción en ambientes virtuales.

La acción social comunicativa asumida en esta investigación, es la conformada por las reglas que rigen socialmente el intercambio comunicativo (Grice, citado en Frías, 2001, p. 16) y los roles sociales de participación, desde:

- La Personalización del mensaje que destine lo que se desea comunicar de manera individual o colectiva con el propósito de fortalecer la bidireccionalidad y multidireccionalidad de la comunicación. Así como el nombre de quien envía el mensaje. Lo anterior permite que los estudiantes tengan una mayor interacción y no se sientan alejados de sus compañeros y el tutor.

- La extensión del mensaje. El texto debe presentar la cantidad de información solicitada en la instrucción, ni más ni menos (Grice citado en Frías, 2001, p. 16). En la comunicación en foros los mensajes muy extensos dificultan el intercambio, al tiempo que el manejo de diversos temas complejiza el análisis y la retroalimentación discursiva.

- La pertinencia del mensaje. Los participantes deben enfocarse al objetivo de aprendizaje en el foro, el tema a tratar con precisión y coherencia, de tal manera, que todos los datos y las argumentaciones, sean elementos que aporten para lograr la meta común (Grice como se citó en Frías, 2001, p. 16). Incluso que aporten sobre lo no dicho, por lo que se recomienda leer las participaciones en el foro antes de elaborar la propia.

- La forma de expresar las ideas. El mensaje en foro debe ser cordial, claro, ordenado en sus expresiones. Se recomienda evitar frases ambiguas que no brinden información literal. Por ejemplo, las frases irónicas o sobreentendidas, chistes, entre otros. Así mismo, el uso de mensajes uniformados en mayúsculas, ya que visualmente son incómodos y pueden interpretarse como una alteración en el estado de ánimo del emisor y/o ausencia de cordialidad.

De la misma manera, es necesario tener en cuenta los roles propuestos por la UNAD, desde su Proyecto Pedagógico Académico Solidario (2008) y que los estudiantes deben asumir para que la interacción sea significativa para el desarrollo de los trabajos colaborativos. El primero de ellos es el rol de Líder, quien debe dinamizar la interacción, debe verificar permanentemente que cada uno de los integrantes de los grupos asuma responsabilidades y que se mantenga un interés por el desarrollo de la actividad. El segundo rol es el de 
Comunicador, es decir, el responsable de la comunicación entre el tutor y el equipo y al mismo tiempo recoge los puntos relevantes encontrados desde la interacción. El tercero es el rol de Relator, quien colabora con la relatoría escrita del proceso de interacción; debe recopilar y sistematizar la información para entregar al finalizar la actividad colaborativa. El cuarto rol es el de Utilero, quien será el responsable de conseguir material adicional al ya contemplado en el curso, y las herramientas de acuerdo a las necesidades del equipo para el desarrollo de las actividades. Finalmente, el Vigía del tiempo, quien será el encargado de controlar el tiempo de los aportes de los compañeros, motivar la participación dentro del cronograma establecido y pactado para la realización del mismo.

\section{Motivaciones}

Es evidente que cuando los estudiantes de la Licenciatura en Inglés como Lengua Extranjera inician su proceso de aprendizaje en la UNAD, tienen diferentes tipos de motivación, algunos tienen una gran influencia externa, en tanto que otros vienen de decisiones propias de los educandos. Haciendo un análisis profundo a lo expresado por los estudiantes en este proceso investigativo, se encontró que hay motivaciones intrínsecas o propias, que no tienen influencia de ningún ente externo y que requieren de la autodeterminación y valores propios, y motivaciones extrínsecas o que cuentan con una marcada influencia externa para realizarse; en el caso de los estudiantes LILE vemos que hay influencias familiares, económicas o simplemente sociales que afectan o benefician sus procesos.

\section{Población y muestra}

El universo poblacional de esta investigación fue conformado por los estudiantes de la Licenciatura en Inglés como Lengua Extranjera de la Universidad Nacional Abierta y a Distancia, que ingresaron entre el segundo periodo académico del año 2011 y el segundo periodo académico de 2012 .

La población total de los tres periodos académicos (2011-2 a 2012-2) fue de 168 grupos de 5 estudiantes cada uno, para un total de 840 estudiantes pertenecientes a la Licenciatura en inglés como Lengua Extranjera; se contó con la participación de 132 estudiantes distribuidos entre los tres periodos académicos, teniendo como muestra el 15\% del total de estudiantes LILE. Los grupos muestra para este estudio investigativo fueron tomados de manera aleatoria en cada periodo académico formal. 


\section{Instrumentos}

En la presente investigación se implementaron instrumentos como:

-Una encuesta virtual de caracterización social, con 28 preguntas, donde se indagó sobre aspectos demográficos, socioeconómicos, familiares, laborales, académicos, de conectividad y de condiciones de contacto o dominio del inglés por parte de los estudiantes.

- Una prueba diagnóstica virtual del dominio de lengua inglesa, con 100 preguntas, diseñada según los diferentes niveles de complejidad de la lengua inglesa (A1-C1) donde se evaluó el dominio de la gramática, comprensión lectora y comprensión auditiva.

-Guía y rúbrica de evaluación del Foro de Reconocimiento del curso (Actividad 2) 551003 - Introducción a la Licenciatura en Inglés como LE.

Es importante tener en cuenta que en el foro de la actividad 2 del curso, se encuentra una guía de actividades donde se explica, paso a paso, que cada estudiante debe actualizar su perfil en el aula y entregar en un archivo Word la respuesta a 4 preguntas, así como una pequeña reflexión sobre los contenidos del curso y sus experiencias previas con el aprendizaje del inglés.

\section{Resultados de investigación}

Los resultados de investigación se presentan desde las tres áreas abordadas en la investigación. En primer lugar, la caracterización general de la población participante, en segunda instancia, los niveles de dominio de lengua inglesa de los estudiantes, y finalmente, las formas de interacción de los estudiantes en el foro de reconocimiento del curso y el análisis de las respuestas dadas a los interrogantes planteados en el mismo.

\section{Caracterización general de los estudiantes}

A continuación se dan a conocer los aspectos más relevantes de la caracterización de los estudiantes nuevos en el programa de Licenciatura en Inglés como Lengua Extranjera de la UNAD, entre los periodos 2011-2 / 2012-2.

En primer lugar, encontramos que la edad de la mayoría de los estudiantes oscila entre los rangos de 16 a 25 años y de 26 a 35 años, Figura 2. 


\section{Estudiantes por rango de edad}

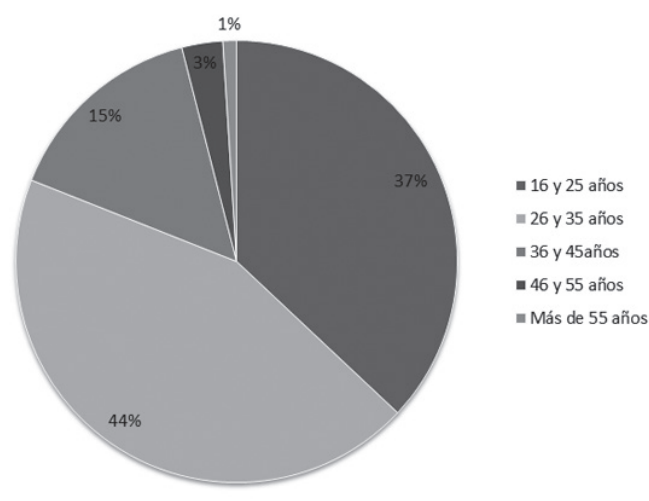

Figura 2. Estudiantes por rango de edad (Fuente: producción propia).

En cuanto al estrato socioeconómico, se puede evidenciar que la mayoría de ellos están ubicados en los estratos 1, 2 y 3, Figura 3 .

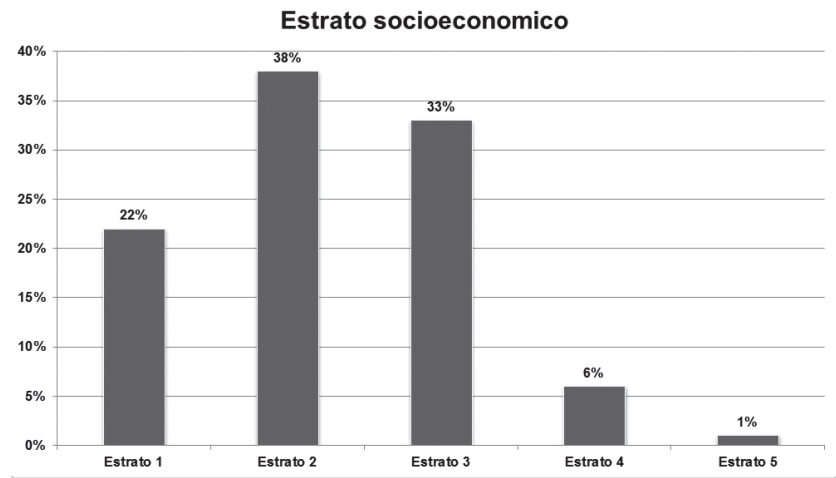

Figura 3. Estrato socioeconómico (Fuente: producción propia).

De la misma manera, encontramos que el 35\% de los estudiantes LILE-UNAD, laboran con una intensidad promedio de 26 a 45 horas semanales, Figuras 4 y 5. 


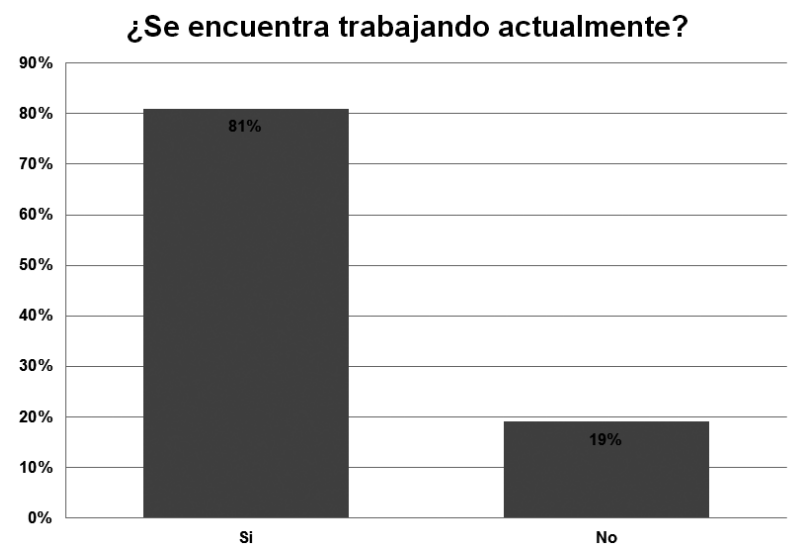

Figura 4. ¿Se encuentra trabajando actualmente? (Fuente: producción propia).

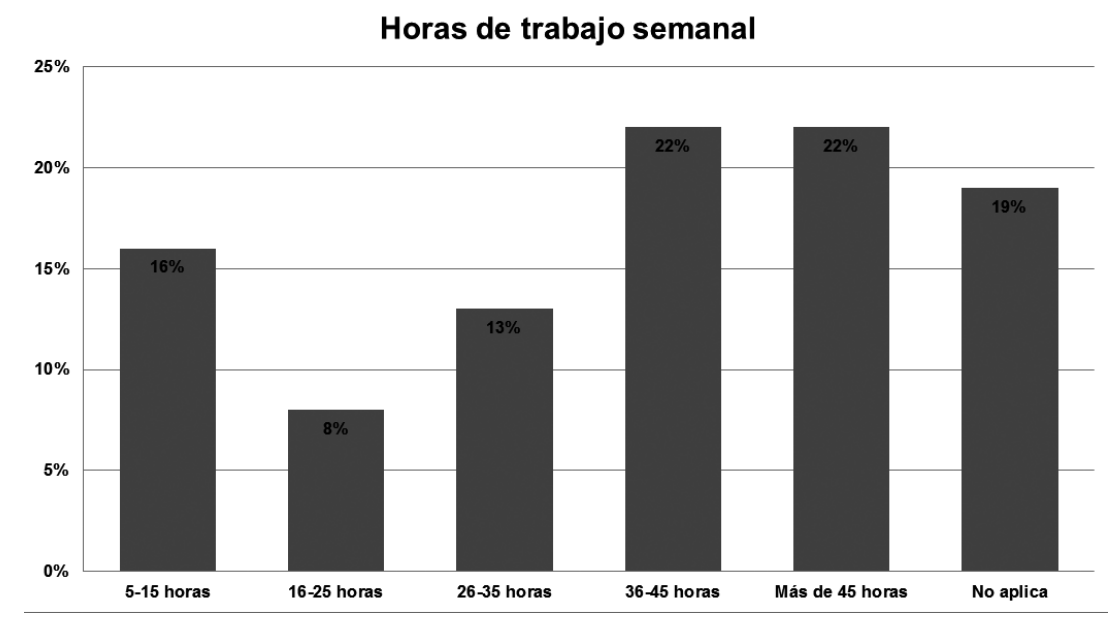

Figura 5. Horas de trabajo semanal (Fuente: producción propia).

También encontramos que el $51 \%$ de los estudiantes han realizado estudios previos pero no los han finalizado; además, la mayoría de ellos (84\%) no han tenido ningún contacto con exámenes clasificatorios en el dominio de la lengua inglesa, Figuras 6 y 7. 


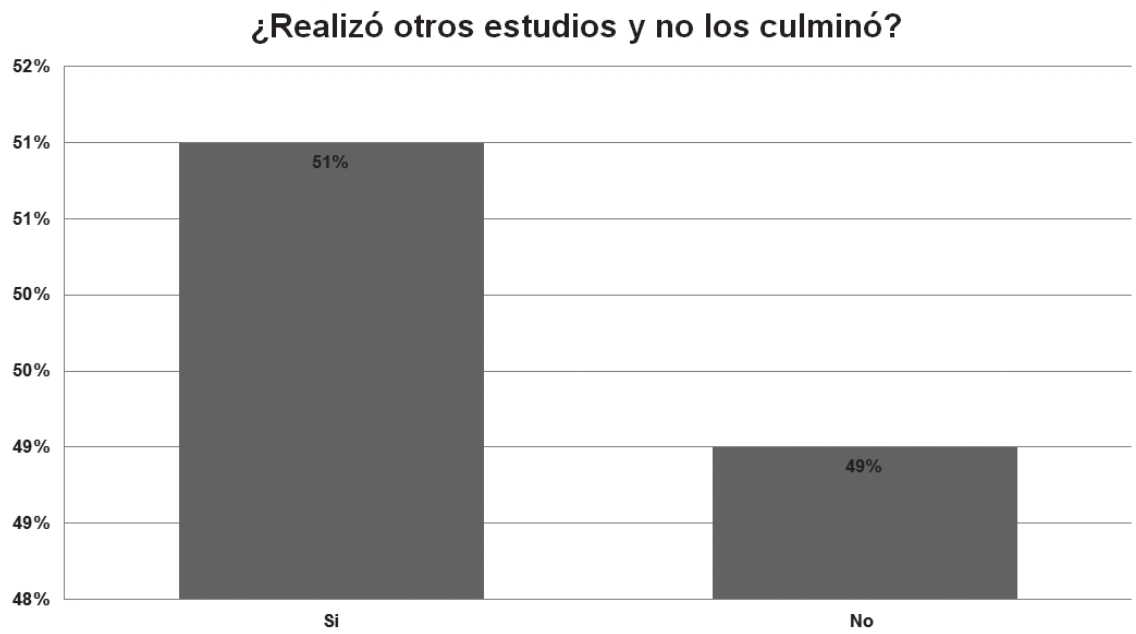

Figura 6. ¿Realizó otros estudios y no los culminó? (Fuente: producción propia).

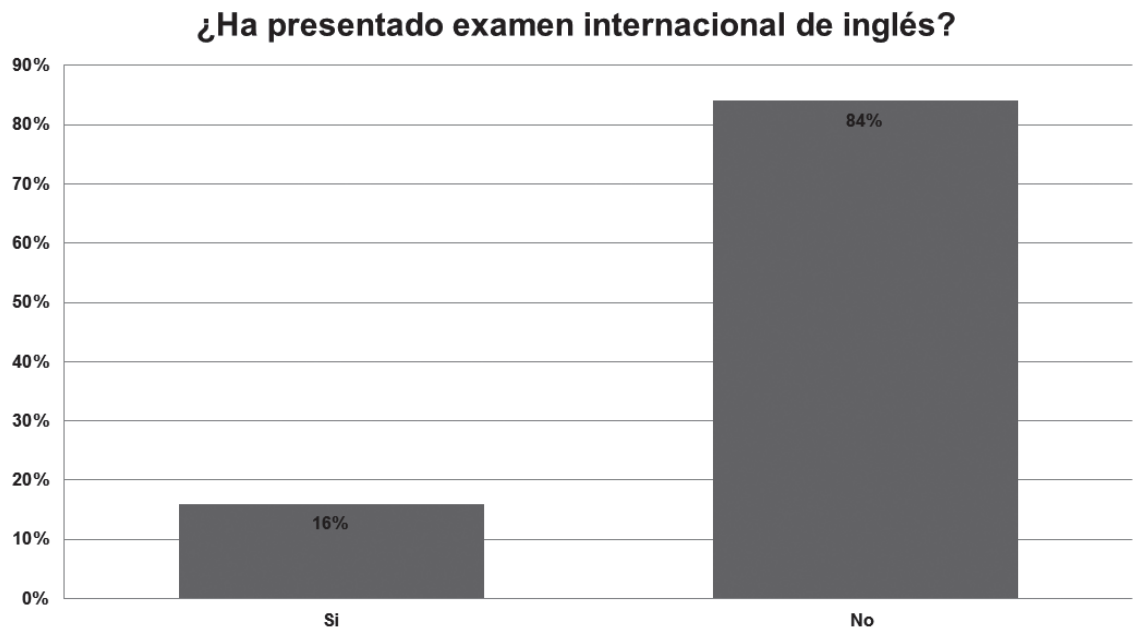

Figura 7. ¿Ha presentado examen internacional de inglés? (Fuente: producción propia).

Como se puede apreciar, la mayoría de los estudiantes LILE-UNAD son jóvenes trabajadores que encuentran en la modalidad distancia-virtual una alternativa para realizar sus estudios formales. Igualmente, ven en la UNAD una posibilidad viable de abordar los costos de sus estudios de acuerdo con su nivel socio-económico. De la misma manera, se evidencia un desconocimiento en las formas de evaluación de sus saberes en el idioma extranjero inglés. 


\section{Niveles de dominio de la lengua inglesa}

Según la prueba realizada a los estudiantes nuevos del programa en los tres primeros periodos académicos, podemos encontrar que la mayoría de ellos, el $89 \%$, cuentan con niveles básicos e intermedios de dominio del inglés y tan solo el $11 \%$ presenta nivel avanzado de manejo del mismo, Figura 8.

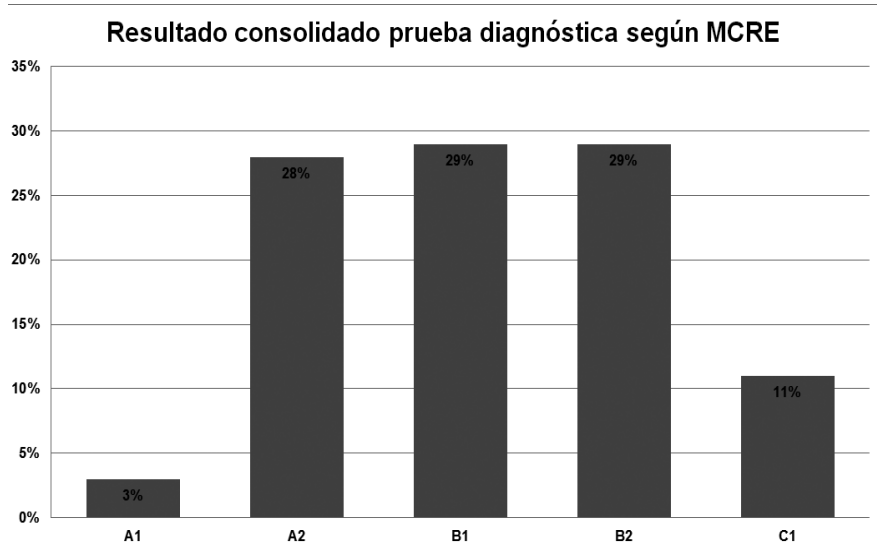

Figura 8. Resultado consolidado prueba diagnóstica según MCRE (Fuente: producción propia).

Lo anterior nos permite concluir que los estudiantes LILE realizan el programa tanto por su profundización en el área de la pedagogía como por su profesionalización docente en el área del inglés.

\section{Formas de interacción en Foro de Reconocimiento}

La presentación de los resultados de los elementos encontrados en la interacción de los foros de reconocimientos se realizó de forma cuantitativa, con un análisis estadístico consolidado entre los tres periodos académicos regulares abordados, según lo propuesto por Camacho et al. (2007, p. 8).

\section{Personalización del mensaje}

Los resultados revelan que el $83 \%$ de los estudiantes muestran en todas sus intervenciones el deseo de recibir realimentación del tutor y los demás integrantes del grupo y se caracterizan por fomentar la integración del grupo haciendo un ambiente amable y comentando el trabajo de los demás. Muchos estudiantes presentan mensajes positivos, algunos de ellos con un saludo muy amable a sus compañeros de grupo y a su tutora; otros aprovechan para contar algo de su vida (en qué ciudad viven, en qué trabajan, su familia); algunos que demuestran una creencia religiosa a la que le confían el éxito del trabajo; otros invitan a los demás a leer su aporte y muestran su interés en conocer los comentarios 
de sus compañeros y aprender de ellos. Hay quienes ven muy positivo todo lo que han hecho sus compañeros y manifiestan la gran expectativa frente a la Universidad y al Programa, mientras hay quienes asignan color a la letra de su mensaje y una imagen que lo haga más amable. Este es el tipo de estudiantes que firman su mensaje con un "Gracias", "Un abrazo", "Dios les bendiga", "Respetuosamente". El 17\% no siempre saluda ni cierra su mensaje con una despedida, tampoco manifiesta siempre lo que espera del grupo ni motiva a leer sus trabajos e interactuar sino que se limita a entregar su aporte. Cuando algunos de ellos comentan el aporte de otro, no escriben a quien se dirigen, solo dan su opinión, por lo que se dificulta saber a quién se refieren. Hay varios que demuestran afán y poca atención al escribir pues no marcan tildes ni signos de puntuación y en ocasiones, aunque dicen que allí entregan su aporte, no hay ningún adjunto.

\section{Extensión del mensaje}

Estos resultados revelan que el $68 \%$ de mensajes adecuados en extensión corresponden a los estudiantes que, sabiendo que el aporte debe estar en un archivo adjunto, por indicación del tutor, le dan a sus mensajes la extensión e información mínima requerida: saludo, presentación del aporte, invitación a leerlo y comentarlo, y despedida. Asimismo, en vista de que los comentarios deben hacerse en el cuerpo del mensaje (no en adjunto), la gran mayoría de estudiantes presenta una estructura estándar que puede definirse como: saludo, comentarios al aporte del compañero y despedida. En general, estos mensajes suelen ocupar máximo 5 líneas. Sin embargo, el 11\% presenta mensajes extensos ya que no siguen la instrucción de entregar el aporte en archivo adjunto, sino que lo desarrollan en la ventana del mensaje. Esto produce mensajes muy extensos (de hasta 20 líneas) lo cual desmotiva la lectura, dificulta la comprensión y genera confusión en los demás integrantes del grupo. Un $21 \%$ corresponde a los estudiantes que redactan mensajes extremadamente cortos que se limitan a decir que ese es su aporte y esperan comentarios, sin una despedida. Otros usan estos mensajes limitados para agradecer algún tipo de observación que se les ha hecho, sin saludar ni despedirse, y unos más hacen un comentario de 4 palabras sobre algún trabajo y se van.

\section{Pertinencia del mensaje}

Según los datos obtenidos, el $60 \%$ de los estudiantes presenta mensajes pertinentes en cuanto a contenido ya que dio respuesta al requerimiento de la actividad. Asimismo, hubo otros que aunque tocaran temáticas diferentes a las que debían tratarse en el aporte, igualmente contribuyeron significativamente al trabajo en grupo. Es el caso de estudiantes que preguntaron sobre aspectos técnicos del aula pues tuvieron dificultad para actualizar su perfil, y quienes compartieron links de interés para todos, como es el caso de una estudiante que referenció un link interesante para aprender inglés en línea y otro que compartió 
uno para motivarse en la enseñanza del idioma inglés. El 19\% entró al foro a participar pocos días antes del cierre, por lo que no desarrolló la actividad con la misma dedicación y atención que los que llevaban más tiempo haciéndolo. El 21\%, correspondiente a los que ingresan el mismo día en que se cierra el foro, se dividen en dos grupos: los que se limitan a entregar su aporte individual sin llevar a cabo un ejercicio grupal, y los que tratan de hacer todo en ese único ingreso: entrega de trabajo y envío de mensajes sencillos a cada uno de sus compañeros, tales como "muy bien" y "te felicito", lo cual no aporta nada profundo ni de calidad al grupo pero lo hacen esperando alcanzar un mayor puntaje.

\section{Forma de expresar las ideas}

Se encontró que el $73 \%$ de los estudiantes presenta claramente sus ideas en al menos un párrafo y corresponde a quienes, generalmente, se toman más tiempo para escribir y ahondar en sus opiniones y aportes; son quienes ingresan al foro con suficiente antelación y dedican más tiempo a la elaboración de la actividad, por lo que logran transmitir, de manera más clara y profunda, sus ideas. Dentro del $27 \%$ se encuentran los que no expresan con claridad su opinión, lo hacen en una línea y participan poco en el foro. Debido a la escasez de palabras, pueden ocasionar ambigüedad, como sucede por ejemplo en los casos donde no se especifica el nombre de la persona a quien va dirigido el comentario.

Es de resaltar que ninguno escribe en mayúsculas sostenidas pues conocen las reglas de netiqueta que se les ha socializado desde el Foro Noticias del curso, lo que refleja una buena apropiación de estas. Igualmente, ninguno hace chistes ni usa frases irónicas o ambiguas hacia sus compañeros y/o tutor. Algunos utilizan colores de fuente diferentes al negro pero nadie usa el rojo, interpretado como agresivo. Asimismo, hay muchos estudiantes bastante participativos que aunque escriben 2 líneas, registran cuatro o cinco ingresos al foro porque destinan cada mensaje a un compañero en particular. Algunos no entregan su aporte en un solo archivo sino que van haciendo avances, por lo que intervienen bastante en el foro hasta completar la entrega de toda la actividad. Estos estudiantes no presentan cada mensaje de manera extensa sino que se limitan a decir a qué punto de la actividad corresponde su aporte y prometen entregar pronto el resto del trabajo.

De manera general, es evidente que los estudiantes no trabajan según los roles definidos por la UNAD para los trabajos colaborativos y nadie toma la vocería para asignarlos. Por el contrario, todos participan, proponen y aportan sin que nadie asuma un liderazgo ni controle el tiempo para la elaboración de la parte individual y grupal. Esto, claramente se ve reflejado en el hecho de que no todos entran a trabajar a tiempo y muchas veces algunos solo lo hacen hasta el último día de plazo. 
Asi mismo, el análisis permite visualizar que la interacción estudiantesdocente en esta muestra es bastante buena ya que el docente es un facilitador del aprendizaje, potencializador de habilidades en el manejo del aula virtual y mediador entre los participantes, por lo que representa un importante factor motivacional en el trabajo grupal. Los docentes de estos grupos dinamizaron el foro, valoraron las contribuciones personales de los estudiantes, promovieron el trabajo en equipo y realizaron un seguimiento personalizado de todos y cada uno de los ellos.

\section{Motivaciones y expectativas de ingreso al programa}

A fin de analizar las motivaciones y expectativas de ingreso de los estudiantes al programa de Licenciatura en Inglés como Lengua Extranjera, se realizaron cuatro preguntas a los estudiantes en el foro del reconocimiento del curso 551003 - Introducción a la Licenciatura en Inglés como Lengua Extranjera.

A continuación se dan a conocer los hallazgos con base en las preguntas realizadas.

\section{Motivaciones de ingreso}

Siendo el Curso Introductorio a la Licenciatura ofertado para los estudiantes de primer periodo académico, es posible evidenciar un entusiasmo generalizado respecto a este programa de formación. Empero, las razones que motivaron a los estudiantes a ingresar a la Licenciatura en Inglés como Lengua Extranjera de la UNAD son diversas, siendo preciso diferenciar las motivaciones intrínsecas (que surgen espontáneamente por satisfacción personal) de aquellas que surgieron de forma extrínseca (influenciadas por razones o situaciones externas).

\section{Motivaciones intrínsecas}

De acuerdo con el análisis realizado, el $68 \%$ de los estudiantes pertenecientes al grupo focal expresan haber tenido motivaciones intrínsecas para decidirse a estudiar la Licenciatura en Inglés como Lengua Extranjera en la UNAD. El gusto por el inglés motivó al 39\% de los estudiantes, la atracción por los idiomas en general fue motivación para el 10\% de ellos mientras que el $7 \%$ de los participantes encontró como motivación el poder fomentar una mejor calidad de vida para sus futuros estudiantes. Encontramos también que el 12\% alude razones tales como: un reto personal, motivación a enseñar inglés debido a las necesidades laborales y académicas existentes, el uso de medios virtuales para el aprendizaje, conocer compañeros y docentes nuevos, entre otros. .

Bajo este panorama, se evidencia que ese $68 \%$ de estudiantes que manifiestan tener motivaciones intrínsecas demuestra que mayoritariamente los estudiantes de LILE ingresaron a este programa por gusto, lo cual, se espera, trascenderá en su éxito académico. 


\section{Motivaciones extrínsecas}

Pero también existen motivaciones extrínsecas recurrentes para ingresar a LILE, fundadas principalmente en el reconocimiento de la importancia del inglés en todos los ámbitos del mundo actual, la ventaja que su dominio representa para la consecución de empleo y el contacto que se ha tenido con la lengua inglesa por diversos motivos, así:

La motivación extrínseca preponderante, que manifestó el 29\% de los participantes, fue el hecho de reconocer la importancia de la legua inglesa hoy en día en el campo laboral, en los negocios y en la vida académica.

Por su parte, el hecho de que esta licenciatura pueda ofrecer más oportunidades de trabajo que otras carreras interesa al 18\%. Este porcentaje de estudiantes encuentra que su futuro como docentes de inglés promete varias alternativas de trabajo, más aun cuando Colombia se proyecta como un país bilingüe para el año 2025.

Otra motivación de este tipo es la experiencia previa que se haya tenido como docente de inglés, pues el $8 \%$ de los participantes comunicaron que han sido profesores de inglés gracias al contacto que han tenido con esta lengua en diversas situaciones; sin embargo se encuentran interesados en adquirir las bases pedagógicas para un mejor enseñanza de la lengua. Igualmente, el inglés como complemento a una carrera ya culminada, el hecho de haber vivido en el exterior o haber tenido un docente admirable por su forma de enseñar la lengua extrajera, fueron motivaciones extrínsecas para el 3\% de la población. El 2\% afirma que decidió ingresar a la licenciatura en inglés por considerar que el dominio de este idioma abre puertas a nuevos conocimientos que no se hallan en nuestra lengua materna. El 1\% afirma haber trabajado en una institución bilingüe que lo motivó a ser parte de esta licenciatura.

Es de resaltar que al analizar tanto las motivaciones extrínsecas como intrínsecas, en general los estudiantes demuestran plena conciencia de que la licenciatura en inglés es un programa cuya finalidad es la de formar docentes; sin embargo, las motivaciones se inclinan en su mayoría por el deseo de aprender y practicar el inglés y el de obtener mayores oportunidades laborales, más que por el gusto hacia la pedagogía o la posibilidad de ser docentes.

\section{Expectativas de Ingreso a LILE-UNAD}

En cuanto a las expectativas de ingreso al programa de Licenciatura en Inglés como Lengua Extranjera, se evidencia que las perspectivas relacionadas con el ámbito académico son menores respecto a las relacionadas con el aprendizaje del inglés en sí, es decir que gran parte de los estudiantes tienen mayor interés por desarrollar las cuatro habilidades en la lengua extranjera que por ser docentes competentes, tal como lo indican los datos a continuación. 


\section{Expectativas relacionadas con el ámbito educativo}

Se evidencia que el $20 \%$ de los participantes centra sus expectativas en convertirse en profesionales competentes. El 17\% se interesa por la adquisición de habilidades en torno a la didáctica del inglés. El 11\% espera aportar al aprendizaje del inglés en su región a través de los conocimientos adquiridos en esta licenciatura. El 10\% busca fomentar una mejor calidad de vida en las comunidades donde viven y, en un sentido similar, el 7\% se interesa por mejorar el nivel y calidad de vida de sus futuros estudiantes, mientras que el $6 \%$ de los docentes en formación esperan ser profesionales útiles a la sociedad. Por otra parte, el $5 \%$ de los participantes desean certificar formalmente la labor que ya desempeñan como docentes de inglés, mientras que el $4 \%$ espera conocer y manejar a futuro los diversos procesos pedagógicos y metodológicos en modalidad virtual, y un mismo porcentaje (4\%) desea formarse como docentes investigadores autónomos. El 3\% de los estudiantes espera poder ejercer la licenciatura en inglés en su municipio y la misma cantidad (3\%) desean formarse como docentes capaces de proponer respuestas creativas a las problemáticas que surgen en la enseñanza del inglés a nivel nacional e internacional.

\section{Expectativas relacionadas con el aprendizaje del inglés}

Las expectativas relacionadas con el aprendizaje del inglés se ubican de la siguiente forma: el $56 \%$ de los participantes espera aprender hablar inglés fluidamente, esto, enfocándose en la parte oral de la lengua. El 73\% de los estudiantes espera aprender inglés perfectamente al desarrollar las cuatro habilidades comunicativas (lectura, escritura, habla y escucha), así como los aspectos gramaticales de la lengua. El 15\% de los participantes desea mejorar las bases previas en el manejo de la lengua inglesa. Respecto a esta misma perspectiva, pero con intencionalidades más específicas, el $8 \%$ de los estudiantes espera adquirir competencias lingüísticas para viajar al exterior, en tanto que el $4 \%$ - que se relaciona con el porcentaje anterior - espera poder comunicarse sin problemas con nativos angloparlantes; el 3\% espera conocer otras culturas e ideologías. El 2\% de los participantes manifiesta el deseo de ayudar a su familia a interactuar con extranjeros, mientras que el $1 \%$ espera poder intercambiar conocimientos con estudiantes angloparlantes.

Comparando las expectativas relacionadas con el ámbito académico, y las expectativas encaminadas al aprendizaje del inglés, sobresale el interés de los estudiantes primordial que los estudiantes por dominar la lengua inglesa. Bajo la Licenciatura en Inglés como Lengua Extranjera de la UNAD debe propender por las condiciones óptimas para la formación de docentes competentes, tanto desde lo pedagógico y didáctico, como desde el manejo adecuado de la lengua inglesa.

\section{Contacto previo con la lengua inglesa}


A continuación se analiza el contacto previo con el inglés que manifestaron haber tenido los participantes del grupo focal, de acuerdo con las categorías de formación académica en inglés y contacto informal con la lengua.

\section{Formación académica en inglés}

En la gráfica anterior, se observa que el $87 \%$ de los estudiantes ha tenido contacto formal con la lengua inglesa desde un ámbito académico; sin embargo el 25\% de los estudiantes afirma haber recibido formación en inglés únicamente en sus instituciones educativas de educación primaria, secundaria y media. El $42 \%$ de los estudiantes ha tomado cursos en diversos institutos de idiomas, y tan sólo el 26\% de ellos los ha culminado. Entre las instituciones más mencionadas se encuentran: el SENA virtual, el Centro Colombo Americano, el instituto Praxis, el Instituto Meyer y el Centro Electrónico de Idiomas. Un 6\% de los participantes ha realizado carreras profesionales con anterioridad, en las cuales el inglés hacía parte del pensum; un $5 \%$ de los estudiantes se encuentran en esta misma posición, pero habiendo culminado una carrera técnica. El 5\% de los participantes ha cursado algunos semestres de licenciaturas en lenguas modernas, pero se han visto en la necesidad de trasladarse a la UNAD debido a su modalidad virtual, lo que les permite manejar diversos turnos laborales y/o estudiar mientras se encuentran fuera del país. El 2\% de los estudiantes manifestaron haber tomado seminarios cortos de inglés; y finalmente, un $1 \%$ mencionó haber estudiado en colegios bilingües o haber recibido un refuerzo de lengua inglesa en el ciclo complementario de su formación como normalista. Bajo este panorama, se evidencia que hay un total de $62 \%$ de estudiantes que han recibido formación en inglés más allá de la que les ofrecieron en la educación primaria, secundaria y media, hecho que lleva a concluir que la mayoría de los estudiantes que ingresan a la licenciatura en inglés como lengua extranjera en la UNAD cuentan con cierto grado de conocimiento de esa lengua, lo que los provee de herramientas para tomar sin mayores dificultades los cursos que ofrece la licenciatura.

\section{Contacto informal con la lengua inglesa}

De acuerdo con los resultados arrojados en esta investigación, un 18\% de los participantes ha tenido contacto con la lengua inglesa fuera del ámbito académico, un 7\% de los estudiantes afirma haber viajado a países angloparlantes tales como Estados Unidos y Canadá. Un 4\% de los participantes ha vivido en un país o región angloparlante: dos de los estudiantes afirman haber vivido en Estados Unidos y dos más viven en San Andrés, en donde su lengua materna es una mezcla entre el creole y el inglés y donde, además, tienen constante interacción con turistas extranjeros que hablan esta lengua. El 3\% de los participantes tiene familiares bilingües cercanos que viven en Colombia, mientras que el $2 \%$ tiene familiares en países angloparlantes con los cuales manifiestan mantener constante interacción. El 2\% de los participantes afirma que su lengua materna es el inglés, 
toda vez que nacieron en Estados Unidos. Sumando el porcentaje de contacto con la lengua inglesa, el de tipo académico llega a un $87 \%$, mientras el contacto informal es de un 18\%. Es posible evidenciar que un 5\% de los estudiantes ha experimentado contacto previo con el inglés tanto desde la formación académica como desde una modalidad informal de las mencionadas.

\section{Conclusiones}

La investigación sobre el perfil del estudiante de la Licenciatura en Inglés como Lengua Extranjera de la UNAD es un excelente insumo para la toma de decisiones académico - administrativas, pues permite, de manera estratégica, seleccionar políticas y prácticas pedagógicas acordes con las características de los estudiantes y de los entornos en donde habitan.

Es indiscutible que el conocimiento que una Institución de Educación Superior tenga sobre la población destino de sus servicios educativos, es un factor preponderante en el desarrollo de políticas que garanticen acciones concretas acordes con el tipo de estudiantes que hacen parte de cada programa de formación académica.

La investigación etnográfica virtual es una excelente metodología para dialogar con los estudiantes desde sus ambientes virtuales de aprendizaje, teniendo en cuenta sus relaciones con la tecnología, sus formas de interacción con la misma y el uso que dan a las herramientas tecnológicas para lograr una comunicación efectiva con sus compañeros y con los propios docentes.

Esta investigación permitió evidenciar que gran parte de la población estudiantil de la Licenciatura en inglés como Lengua Extranjera ha ingresado al programa debido al gusto por la lengua inglesa y a la importancia que ella tiene en el campo laboral; sin embargo, el interés por la docencia no es un factor primordial en la escogencia del programa académico. Por este motivo, uno de los retos de esta licenciatura consiste en concienciar a los futuros docentes respecto a sus objetivos de formación y proyección profesional, con miras al fortalecimiento no sólo de las competencias en torno al dominio del inglés, sino también de aquellas que tienen que ver con su formación pedagógica y didáctica. Para ello, es fundamental acercar a los estudiantes a escenarios educativos formales desde sus primeros periodos académicos, así como afianzar los procesos propios de la práctica pedagógica del programa, dando respuesta así a la necesidad que presenta nuestro país respecto a la formación de docentes de inglés idóneos en sus diversos ámbitos de actuación.

Por otra parte, este estudio permitió reiterar la pertinencia del programa LILE de la UNAD en el contexto colombiano, ya que un gran porcentaje de estudiantes encontró en este programa la oportunidad de prepararse profesionalmente sin tener que abandonar su trabajo u otras obligaciones que no les permiten acceder 
a la educación superior en modalidad presencial. En situaciones similares se encuentran los estudiantes que viven en regiones alejadas del país, donde no llegan otras opciones de formación profesional, además de las que les ofrece la UNAD, razón que los motiva a educarse de forma comprometida con la finalidad de transformar sus comunidades, mejorando la calidad de vida y las oportunidades de desarrollo de las mismas a través de su futura labor docente.

\section{Referencias bibliográficas}

Barberá, E. Badia, A. Mominó de la Iglesia, J. 2001. Enseñar y aprender a distancia: ¿es posible? Universitat Oberta de Catalunya. Recuperado el 11 de junio de: http://www. uoc.edu/web/esp/art/uoc/0105018/ensapren.html

Calero, A. Calero, L. 1989. Plan de investigación del perfil del estudiante universitario colombiano. Bogotá: Oficina de Planeación y Desarrollo Institucional.

Camacho, C. Pacheco, A. Pereida, M. 2007. "El análisis e interpretación de las interacciones en los foros virtuales: descripción de parámetros orientadores para mejorar la calidad". En: Virtual Educa Brasil 2007. Recuperado el 24 de abril de 2013 de: http://reposital.cuaed.unam.mx:8080/jspui/bitstream/123456789/1635/1/206-CCR. PDF

Frías, X. 2001. Introducción a la pragmática. Recuperado el 27 de noviembre de 2013 de: http://www.romaniaminor.net/ianua/sup/sup05.pdf

Grice, P. (s.f.) "La búsqueda de significado". En: Lecturas de Filosofía del Lenguaje. Luis Valdes Editor. 2da. Edición. Recuperado el 23 de mayo 2013 de: http://www. textosenlinea.com.ar/academicos/Grice\%20-\%20Logica\%20y\%20conversacion.pdf

Hine, C. 2000. Virtual Ethnography. London: SAGE Publication Ltd.

Mason, B. 2001. Issues in virtual ethnography. Recuperado 10 julio de 2012 de: http:// www.ucs.mun.ca/ bmason/pubs/lime99.pdf

Ministerio de Educación Nacional. 2006. Estándares Básicos de Competencias en Lenguas Extranjeras. Serie Guías No. 22. Recuperado 8 de junio de 2013 de: http:// www.colombiaaprende.edu.co/html/mediateca/1607/articles-115375_archivo.pdf

Moore, M. Anderson, W. 2003. Handbook of distance education. London: Lawrence Erlbaum Associates Publisher. Recuperado el 12 de noviembre de 2013 de: http://www.uady.mx/ contadur/sec-cip/articulos/libros_online/educacion/ LawrenceErlbaum2003HandbookOfDistanceEducation.pdf 
Sánchez, A. Puerta, C. Sánchez, L. 2010. Manual de comunicación en ambientes educativos virtuales. Medellín: Fundación Universitaria Católica del Norte. 256 p. Recuperado el 6 de octubre de 2013 de: http://www.ucn.edu.co/institucion/salaprensa/Paginas/Publicaciones/manual-comunicacion-ambientes-virtuales.aspx

Universidad Nacional Abierta y a Distancia - UNAD. 2012. 551003 - Curso Introducción a la Licenciatura en Inglés como Lengua Extranjera. Bogotá: UNAD. 